\title{
A comparative study of thermal and acoustical insulations used for residential buildings
}

\author{
Rachid Lagtayi ${ }^{1,3^{*}}$, Lamya Lairgi ${ }^{2}$, Ahmed Khouya ${ }^{3}$ and Abdelmajid Daya ${ }^{2}$ \\ ${ }^{1}$ Department of Sciences, Industrial and Civil Technologies, Team of sciences and advanced \\ technologies, National School of Applied Sciences, Abdelmalek Essaadi university, 2222 Tetouan, \\ Morocco \\ ${ }^{2}$ Department of Physics, Laboratory of M3ER-, FSTE, Moulay Ismail University, 509 Errachidia, \\ Morocco \\ ${ }^{3}$ Department of Electrical and Industrial Engineering, National School of Applied Sciences, 1818 \\ Tangier, Morocco
}

\begin{abstract}
The aim of this paper is to study the thermal and acoustical insulation of four different buildings' envelopes mostly used for residential buildings in Tetouan city. The thermal and the acoustical models were investigated during summer season, while a linear nonmonochromatic source of sound has been placed $1 \mathrm{~m}$ away from the exterior side of each wall and it is emitted a low-frequency sound waves with a velocity of $200 \mathrm{~m} / \mathrm{s}$. The study shows that the double layers of hollow clay brick wall with a medium of air gap presents a good thermal resistance and soundproofing compared to the other walls under study, due to the insulation by air gap with a thickness of $0.06 \mathrm{~m}$. Moreover, the homogenous and thick walls are simultaneously performant as thermal and sound insulators.
\end{abstract}

\section{Introduction}

Nowadays, the energy consumption is one of the most important topics for building sector, whereas, in Morocco, the construction sector constitutes $25 \%$ of the total consumed energy, of which $18 \%$ for residential and $7 \%$ for the tertiary buildings [1].

The thermal insulation of buildings strongly affects the energy consumption, due to its vital role in regulating the temperature between the indoor and outdoor environments, While, reducing and preventing the inward noise through the buildings' envelope is also needed for realizing simultaneously thermal and acoustic comfort $[2,3]$.

Consequently, it is important to choose the appropriate thermal insulators for realising buildings' thermal comfort and minimize the energy consumption of the Heating, ventilation, and air conditioning (HVAC) system. Otherwise, the acoustical insulation is also required due to the increase of the environmental noise [4, 5], populations density growth, development of industry, transport, and aviation [6], while the most buildings does not have soundproofing products which is constantly demanded [7]. The acoustical insulation performance is strongly dependent on the physical characteristics of the used materials, observer positions, buildings envelopes design and acoustical properties [8-10].

The aim of this work is to present a comparative analysis of thermal and acoustical insulations mostly used for residential buildings in Tetouan city, Morocco. This study will

* Corresponding author: rachid.lagtayi1@etu.uae.ac.ma 
be helpful to find the right models to realise simultaneously the thermal and acoustical comfort, saving energy, and conserve the safety of our environment.

\section{Model description}

In this study, we have chosen four different buildings envelopes mostly used in Tetouan, Morocco. Tetouan is located in the north of morocco and it is characterized by a Mediterranean climate. The yearly average temperature in Tetouan is $18.7^{\circ} \mathrm{C}$ and the average precipitation is $600.7 \mathrm{~mm}$. The figure 1 presents the average monthly temperatures in Tetouan, Morocco.



Figure. 1. Average monthly temperatures in Tetouan, Morocco.

Figure 2 shows the four types of the studied buildings envelopes which are: A single layer of hollow clay brick wall (Wall(a)), a single layer of hollow concrete brick wall (Wall(b)), double layers of hollow clay brick wall with a medium of polystyrene (Wall(c)), and double layers of hollow brick wall with a medium of air gap (Wall(d)).
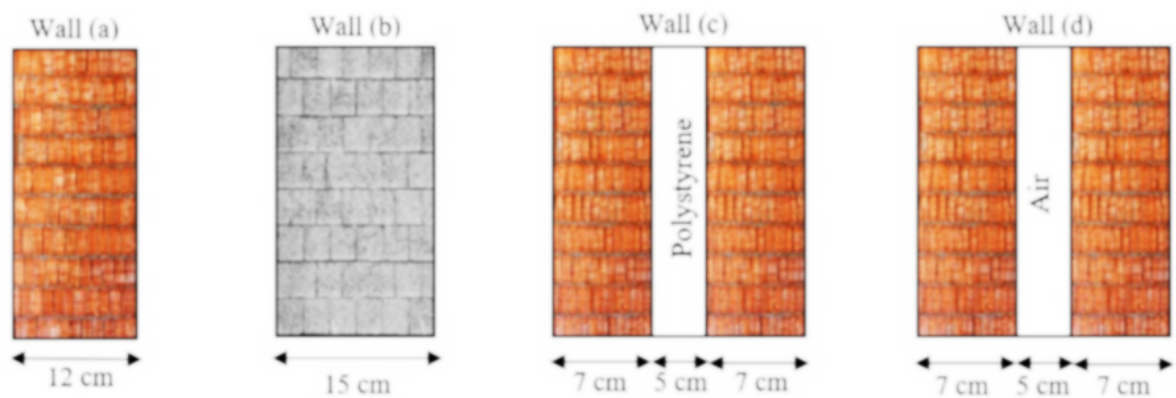

Fig. 2. External buildings' envolopes under study.

Figure 3 shows the proposed model for studying different acoustic insulators as it is shown below: 


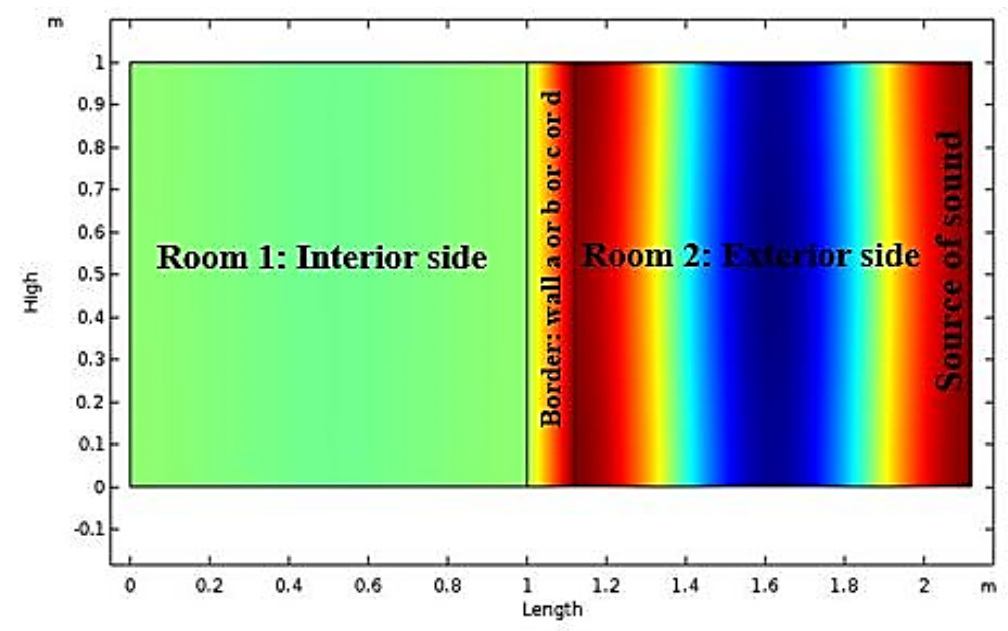

Fig. 3. Acoustical model

\section{Results and discussions}

Figure 4 presents the temperature variations through the walls (a), (b), (c) and (d) on the $7^{\text {th }}$ of August 2019 at 2 p.m, while the outside environment temperature was $37^{\circ} \mathrm{C}$ (summer season). the outer and the inner facades' temperatures change respectively from $32.95{ }^{\circ} \mathrm{C}$ to $28.15^{\circ} \mathrm{C}$ for wall (a), from $33^{\circ} \mathrm{C}$ to $29.7^{\circ} \mathrm{C}$ for wall (b), from $34.75^{\circ} \mathrm{C}$ to $23.85^{\circ} \mathrm{C}$ for wall (c) and from $34.80^{\circ} \mathrm{C}$ to $23.45^{\circ} \mathrm{C}$ for wall (d), which shows that the temperature variations from the outer facade toward the inner facade are decreased by $14.6 \%$ for the wall(a), $10 \%$ for the wall (b), 31.4\% for the wall (c) and $32.6 \%$ for the wall (d), which explains that the walls (a) and (b) have a weak ability to reduce heat transfer trough them, while the wall (d) presents a good thermal resistance in comparison with the other walls under study because of the insulation by air gap with a thickness of $0.06 \mathrm{~m}$, which gives it a good capacity to block heat dissipation and conserve heating during winter season, moreover, this result is the same as that found by others researchers [11].

Otherwise, the figure 5 shows the change of the sound pressure level while sound waves cross the walls (a), (b), (c) and (d). We note that the sound pressure level is reduced by $20.4 \%$ through the wall (a), 21.4\% through the wall (c), 31.6\% through the wall (b) and $36.7 \%$ through the wall (d). This result shows that the wall (d) presents the highest rate of sound pressure attenuation in comparison with the other walls under study. The walls (a) and (c) are characterized by the lowest rate of sound pressure attenuation in comparison with the other walls, because the wall (a) is thin compared to other walls and the wall (c) isn't homogenous compared to the walls (b) and (d).

We deduce that the thermal/acoustic insulation of wall (d) is performant compared to the other walls under study, due to the insulation by air gap and we note also that homogeneous and thick walls constitute a good correlation for thermal and acoustical insulation in our case study, but the thermo-physical properties of the used materials could affect negatively these two characteristics. Despite this, the correlation will be a good solution for realizing simultaneously energy savings and acoustical comfort. 


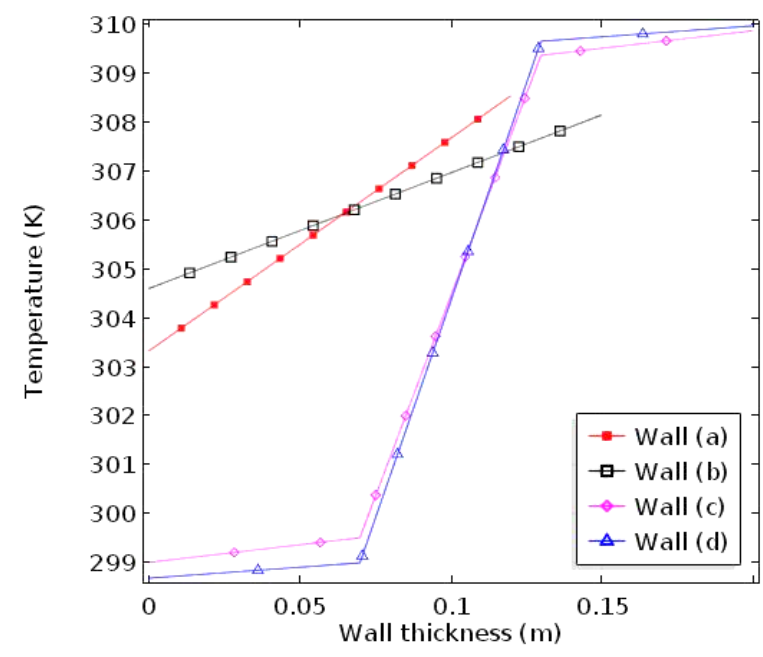

Fig. 4. Temperature variation in terms of the thickness of the walls (a), (b), (c) and (d).

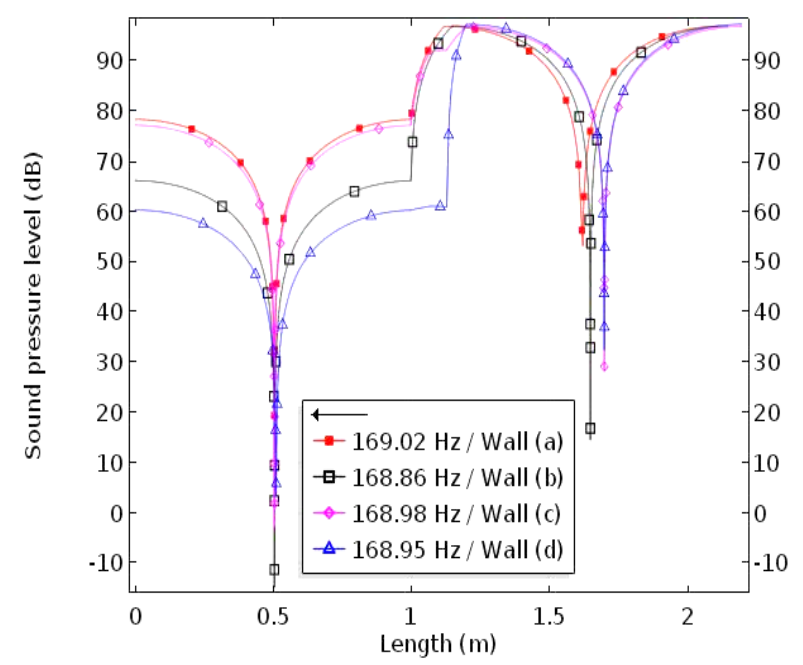

Fig. 5. Sound pressure Level variation across the walls (a), (b), (c), and (d).

\section{Conclusion}

In this work, we conclude that despite the performant thermal insulation of certain buildings envelopes, their acoustic insulation performances could be very weak, which could negatively influence the acoustic comfort of inhabitants, while the homogenous and thick walls as walls (b) and (d) have proved a good ability to reduce noise transmission in comparison with walls (a) and (c), but their acoustical insulation performances still not good enough.

Finally, choosing the efficient thermal/acoustic insulators still important to ensure a good thermal and acoustic comfort for inhabitants but the correlation between these two characteristics still quite difficult, while finding correlated models for homogenous and thick walls of similar structure is possible.

\section{Acknowledgements}

We would like to thank every single author for her/his contribution to achieve this work.

\section{References}

1. AMEE, Règlement thermique de construction au Maroc (RTCM), (2013).

2. H. M. Lee, Y. Liu, H. P. Lee, Assessment of acoustical environment condition at urban landscape. Applied Acoustics. 160, 107126 (2020).

3. C. Zou, R. Zhu, Z. Tao, D. Ouyang, Y. Chen, Evaluation of Building ConstructionInduced Noise and Vibration Impact on Residents. Sustainability. 12, 1579 (2020).

4. Ö. Gürsoy, N. Y. Akdağ, Evaluation of the environmental noise problems in holiday villages: a case study from Antalya, Turkey. Environmental Science and Pollution Research. 26, 10972-10986 (2019).

5. M. K. Nematchoua, J. C. V. Noelson, I. Saadi, H. Kenfack, A.-Z. F. R. Andrianaharinjaka, D. F. Ngoumdoum, J. B. Sela, S. Reiter, Application of phase change materials, thermal insulation, and external shading for thermal comfort 
improvement and cooling energy demand reduction in an office building under different coastal tropical climates. Solar Energy. 207, 458-470 (2020).

6. M. Sørensen, T. Münzel, M. Brink, N. Roswall, J. M. Wunderli, M. Foraster, in Advances in Transportation and Health (Elsevier, 2020), pp. 105-131.

7. D. Bard, N.-G. Vardaxis, E. Sondergard, Acoustic Comfort Investigation in Residential Timber Buildings in Sweden. J. Sustain. Archit. Civ. Eng. 1, 78-89 (2019).

8. A. Taghipour, T. Sievers, K. Eggenschwiler, Acoustic comfort in virtual inner yards with various building facades. International journal of environmental research and public health. 16, 249 (2019).

9. M. Matheou, A. Couvelas, M. C. Phocas, Transformable building envelope design in architectural education. Procedia Manufacturing. 44, 116-123 (2020).

10. B. Xue, L. Xie, Y. Bao, J. Zhang, Multilayered epoxy/glass fiber felt composites with excellently acoustical and thermal insulation properties. Journal of Applied Polymer Science. 136, 46935 (2019).

11. L. Lairgi, A. Daya, R. Elotmani, M. Touzani, Contribution to the study of the energy needs for a building in the city of errachidia influence of orientation and local materials. Key Engineering Materials. 820, 18-28 (2019). 